3 Miwa K, Kambara H, Kawai C. Variant angina aggravated by aspirin. Lancet 1979;2:1382

4 Habbab MA, Szwed SA, Haft JI. Is coronary arterial spasm part of the aspirin-induced asthma syndrome? Chest 1986;90:141-3

5 Kontos MC, Jesse RL, Tatum JL, Ornato JP. Coronary angiographic findings in patients with cocaine-induced chest pain. J Emerg Med 2003;24:9-13

\section{Splenomegaly, anaemia and pleural effusion}

\author{
P Kojodjojo MRCP S Chew BSc \\ $\mathrm{K}$ Wakeham BSc MRCP R Lancaster FRCP
}

J R Soc Med 2005;98:23-24

Most pseudocysts of the spleen cause no symptoms, but occasionally they lead to disaster.

\section{CASE HISTORY}

A man of 71 was admitted after syncope. He was on treatment for hypertension, atenolol $25 \mathrm{mg}$ daily, with good control. Recently he had lost a few kilograms but had otherwise been fit and well. He had not travelled abroad for many years and was teetotal.

His blood pressure was $133 / 76 \mathrm{mmHg}$ without postural drop, pulse $78 / \mathrm{min}$. On chest examination there was a small area of stony dullness at the left base, consistent with a small pleural effusion; the abdomen was distended and a very large spleen could be felt. Investigations revealed a normochromic normocytic anaemia (haemoglobin $10.3 \mathrm{~g} /$ $\mathrm{dL})$, white cell count $13.7 \times 10^{9} / \mathrm{L}$ (81\% neutrophils, $17 \%$ lymphocytes) and platelets $307 \times 10^{9} / \mathrm{L}$. Liver function tests, corrected calcium and clotting profile were normal. Blunting of the left costophrenic angle was seen on a chest radiograph, in keeping with the positive respiratory findings. Further investigations were requested including serum electrophoresis, hepatic screen, abdominal imaging and bone marrow studies.

Several hours later the patient experienced presyncope on the ward and blood pressure declined to $97 / 62 \mathrm{mmHg}$, pulse $74 / \mathrm{min}$. Clinically, the left-sided pleural effusion was larger and the haemoglobin was now $8.1 \mathrm{~g} / \mathrm{dL}$. The abdomen remained non-tender and the size of the spleen had not obviously changed. A diagnostic pleural tap drew a

Department of General Medicine, St Mary's Hospital, London W2 1NY, UK Correspondence to: Pipin Kojodjojo, Waller Department of Cardiology, St Mary's Hospital, Praed Street, London W2 1NY, UK

E-mail: pipin.kojodjojo@imperial.ac.uk

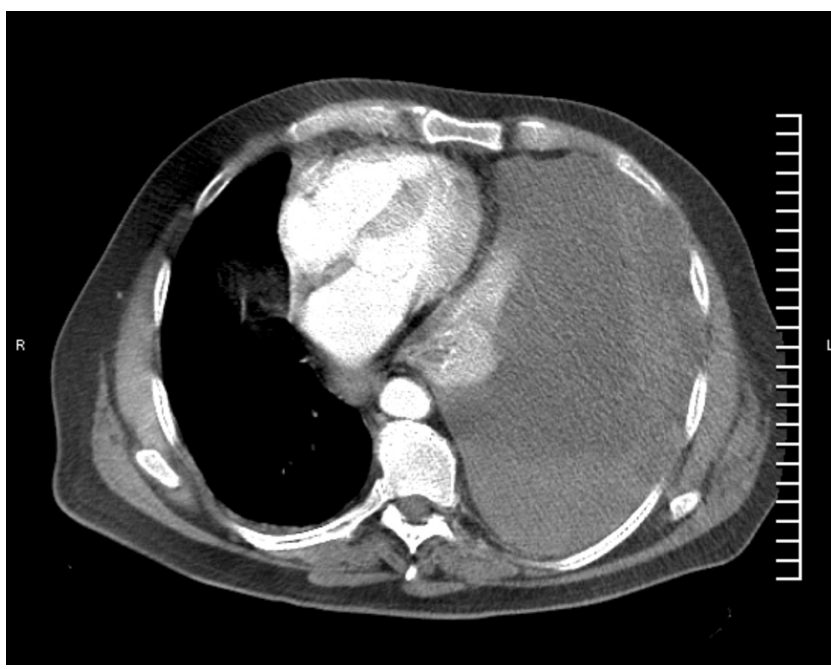

Figure 1 CT of chest showing large left pleural effusion around the collapsed lung (black arrow) with associated mediastinal shift

small amount of dark blood. Central venous and urinary catheters, along with an arterial line, were inserted to monitor his haemodynamic status more closely. CT showed a large effusion almost completely filling the left hemithorax, associated with complete left lung collapse and mediastinal shift (Figure 1). The spleen was massively enlarged, measuring $23 \times 23 \times 17 \mathrm{~cm}$ - a cyst with only a thin rim of splenic tissue (Figure 2). No rib fractures, other bony injuries or lymphadenopathy were seen.

His blood pressure responded to blood and colloid transfusions, but before he could be transferred for exploratory thoracolaparotomy he had a cardiac arrest. After restoration of the pulse and fluid administration, insertion of an intercostal drain yielded $3 \mathrm{~L}$ of dark altered blood. At operation he was found to have a splenothoracic fistula. There was no blood in the peritoneal cavity. The spleen was removed and the left diaphragm was repaired.

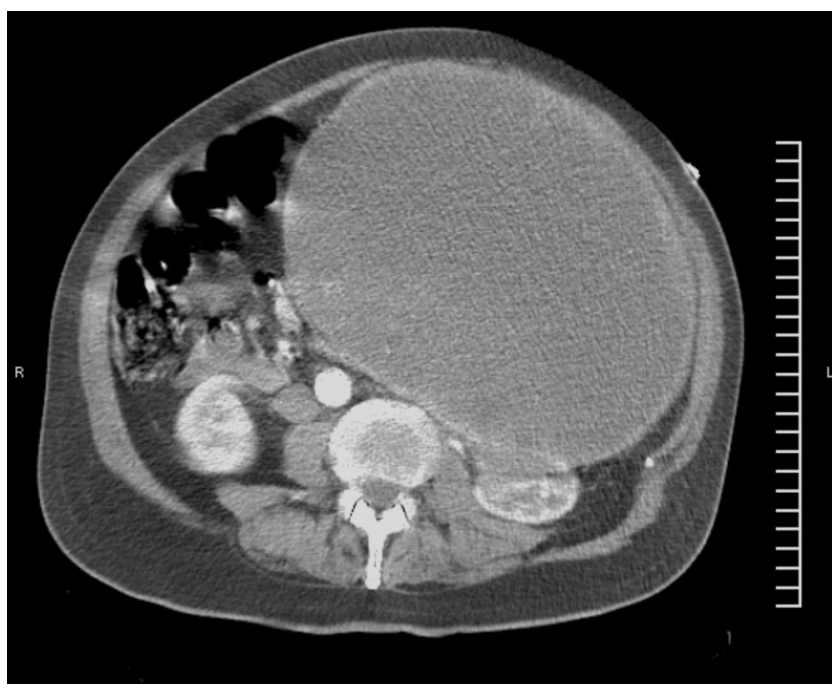

Figure $2 \mathbf{C T}$ of abdomen showing thin rim of splenic tissue encasing cyst 
On sectioning, the spleen contained a large fibrous-walled pseudocyst filled with altered blood and clots. One pole was adherent to part of the left hemidiaphragm. Postoperatively the patient succumbed to acute respiratory distress syndrome.

\section{COMMENT}

The most likely differential diagnoses for a man of this age with massive splenomegaly associated with anaemia, unilateral pleural effusion and weight loss are a myeloproliferative or lymphoproliferative disorder, portal hypertension in the context of cirrhotic liver disease, malignancy and infiltrative conditions such as amyloidosis. Chronic infections such as brucellosis could present in the same way but were unlikely in the present case because of the lack of travel outside the UK.

The probable train of events was haemorrhage into the pseudocyst and fistulation into the left hemithorax. The absence of tachycardia after syncope may be explained by his treatment with a beta-blocker.

Splenic cysts can be classified as primary or secondary (pseudocyst). Pseudocysts constitute 56\% of all nonparasitic splenic cysts and are mostly of traumatic origin. ${ }^{1}$ Indeed, the spleen is the intra-abdominal organ most commonly injured by blunt abdominal trauma and up to a quarter of patients with blunt splenic trauma sustain pleuropulmonary complications (lung contusion, pleural effusion). ${ }^{2}$ Our patient was unable to recall any previous trauma and witnesses reported none during his presenting syncopal episode.

Splenic pseudocysts are commonly silent but can be complicated by abscess formation and rupture, leading to acute abdomen and haemoperitoneum. ${ }^{2}$ Rupture into the chest has been recorded in blunt splenic trauma, hydatidosis, angiosarcoma and chronic myeloid leukaemia. ${ }^{3-6}$ Spontaneous rupture of a pseudocyst, with a splenothoracic fistula and haemothorax, is clearly very unusual-especially in the absence of a haemoperitoneum.

\section{REFERENCES}

1 Economides NG, Benton BF, Fortner TM, et al. Splenic pseudocysts: report of two cases and review of the literature. Am Surg 1980;46:644-8

2 Gorenstein A, Witzling M, Haftel LT, et al. Pleuro-pulmonary involvement in children with blunt splenic trauma. J Paediatr Child Health 2003;39:282-5

3 De Waele JJ, Vermassen FE. Splenic herniation causing massive haemothorax after blunt trauma. J Accid Emerg Med 1999;16:383-4

4 Kuo YC, Wang HC, Chu KA, et al. Chronic myeloid leukemia initially presenting with spontaneous mediastinal hematoma and hemothorax. Zhonghua Yi Xue Za Zhi (Taipei) 2000;63:170-4

5 Millan J, Rodriguez P, Gallego P, et al. Spontaneous splenic rupture with hemothorax without hemoperitoneum. Description of a case of angiosarcoma of the spleen. Rev Clin Esp 1988;183:334-5

6 Venissac N, Alifano M, Mouroux J. Splenic hydatidosis complicated by a splenothoracic fistula: report of a case. Surg Today 2002;32:1023-5

\section{Thrombotic endocarditis}

\author{
Kate M Evans BM MRCP \\ Heather E Robinson BM MRCP \\ Patrick K Chong MD MRCP
}

J R Soc Med 2005;98:24-25

Non-bacterial thrombotic endocarditis is a complication of acute disorders such as septicaemia and burns, but the most common underlying condition is malignant disease.

\section{CASE HISTORY}

A woman of 55 came to hospital after an acute confusional episode, preceeded by ten days of lethargy, anorexia and chest and abdominal pains. She had a history of hypothyroidism and vitiligo, and was taking thyroxine.

On examination she was alert and afebrile. The only noteworthy finding was mild suprapubic tenderness. Her neutrophil count was $12.2 \times 10^{2} / \mathrm{L}$ and C-reactive protein was high $(299 \mathrm{mg} / \mathrm{L})$. When ward urinalysis proved abnormal she was started on levofloxacin for a presumed urinary tract infection. Subsequently, the chest radiograph showed a right upper lobe wedge-shaped opacification, felt to be consistent with tumour, consolidation or infarction. CT of the thorax was therefore arranged. Forty-eight hours later she became feverish and confused and developed a dense left hemiparesis. CT of the head revealed two separate infarcts, in the right middle cerebral artery territory and the right cerebellar hemisphere. There were no features of cerebral metastases. When a transthoracic echocardiogram revealed no cardiac source of emboli, transoesophageal echocardiography was arranged, but two days later the patient deteriorated, with a fall in the level of consciousness and development of a right hemiparesis. Repeat CT of the head now revealed a third infarct, in the left middle cerebral artery territory. Total body CT showed extensive pulmonary consolidation of the right upper lobe, thrombus in both right and left pulmonary arteries, and multiple renal and splenic infarcts, but no evidence of malignancy.

The differential diagnosis included infective endocarditis with septic emboli and a systemic vasculitis. For the former possibility she was treated with intravenous benzylpenicillin and gentamicin, and for the latter she received three pulses of intravenous methylprednisolone. There was no neurological improvement and subsequent immunological

Derriford Hospital, Plymouth PL6 8DH, UK

Correspondence to: Dr K M Evans, c/o Dr Chong's secretary, Level 9, Derriford Hospital, Plymouth PL6 8DH, UK

E-mail: kme@doctors.org.uk 